\title{
Synthèse de l'atelier "Migrations et réfugiés"
}

Report of Workshop "Migrations and Refugees"

Danielle de Lame et Win Van Binsbergen

\section{(2) OpenEdition}

\section{Journals}

Édition électronique

URL : http://journals.openedition.org/apad/176

DOI : $10.4000 / a p a d .176$

ISSN : 1950-6929

Éditeur

LIT Verlag

Édition imprimée

Date de publication : 1 décembre 2002

Référence électronique

Danielle de Lame et Win Van Binsbergen, "Synthèse de l'atelier "Migrations et réfugiés" », Bulletin de I'APAD [En ligne], 23-24 | 2002, mis en ligne le 15 décembre 2006, consulté le 23 septembre 2020.

URL : http://journals.openedition.org/apad/176 ; DOI : https://doi.org/10.4000/apad.176

Ce document a été généré automatiquement le 23 septembre 2020

Bulletin de l'APAD 


\title{
Synthèse de l'atelier "Migrations et réfugiés"
}

\author{
Report of Workshop "Migrations and Refugees"
}

Danielle de Lame et Win Van Binsbergen

\section{NOTE DE L'ÉDITEUR}

Rapporteur : Danielle de Lame. Discutant : Win Van Binsbergen

1 Au regard du thème général du colloque, les relations entre les services publics et leurs usagers, les interventions au cours de cet atelier mettent l'accent sur les usagers de services très particuliers en ce sens que l'offre de ces services détermine des statuts exceptionnels et crée des conditions particulières, souvent temporaires, d'accès à ces services.

Pour ce qui est des réfugiés, cette affirmation semble claire. Les grands organismes humanitaires interviennent dans des situations de crise. L'octroi du statut de réfugié définit les conditions d'accès à des ressources temporaires dans un espace redéfini, à la fois par la situation de conflit et par l'installation des réfugiés. Ce qui est rarement étudié, ce sont les interactions de ces réfugiés, de ces camps, avec les populations où les camps sont implantés ou encore, au retour, les effets de la situation d'ex-réfugié au retour dans la région d'origine.

En ce qui concerne les migrations individuelles, le sens de cette affirmation est moins clair. Et pourtant... Le migrant, lui aussi, en appelle à des registres identitaires divers et capitalise un acquis lié au passage de frontières, un acquis qu'il tente de pérenniser dans son milieu d'insertion permanente. La mise en perspective des différents cas de figure engage une réflexion sur la création des espaces, la projection de l'organisation sociale sur le territoire, la ré-appropriation durable d'infrastructures temporaires. 
Voyons maintenant les thèmes communs sous-jacents à la diversité des présentations orales de cet atelier. Nous les situerons, en conclusion, dans la perspective plus générale du colloque.

Les migrations transfrontalières, y compris lorsqu'elles se produisent entre l'espace artificiellement défini du camp (de déplacés si les "réfugiés" le sont à l'intérieur de leur territoire national) et le "hors camp", créent des plus-values liées au franchissement des frontières (en particulier lorsque les règles internationales ne respectent pas les normes d'éloignement des camps par rapport à la région d'origine, ce qui est fréquent). Ce franchissement crée des statuts particuliers, statut officiel de réfugié lié aux règles internationales, statut d'étranger, mais aussi statut d'intermédiaire dans l'accès à des ressources inhabituelles. Les références territoriales sont élargies et on assiste à un pluralisme des enracinements territoriaux et juridiques de référence. Une plus-value résulte $\mathrm{du}$ franchissement des frontières. Le recours à des références identitaires démultipliées, dont certaines sont transnationales (les réfugiés, les Mourides, les Touaregs, etc.) sert de passeport social. Les habitudes de la société d'origine structurent les nouveaux rapports à l'espace, que ce soit dans la projection de l'organisation sociale d'origine sur le nouvel espace d'insertion, ou dans la création de réseaux. Une fois revenus à une situation stable, migrants et réfugiés tentent de pérenniser les acquis de leur expérience en fonction des modes d'organisation de leur société d'origine. Les stratégies individuelles et collectives s'y conjuguent d'une façon analogue à ce qui se produit dans tous les cas où des ressources exceptionnelles sont mises à la disposition d'une communauté.

6 La présentation de Laurence Touré illustre bien l'ensemble de la problématique. En effet, elle montre comment, dans le contexte des conflits et des déplacements importants de populations au nord du Mali, la situation d'encadrement dans des camps de réfugiés et de déplacés - les dépendants agricoles ayant pris des destinations souvent différentes de celles des pasteurs - a induit une plus grande capacité à percevoir des opportunités nouvelles - notamment les avantages de proximité liés aux infrastructures - et à les capter. Cette adaptabilité a permis à certains leaders des camps d'amasser un capital relationnel et matériel qui a modifié les équilibres sociaux au retour. Le retour s'effectue dans un milieu transformé. Dans une situation de précarité écologique, certains leaders touaregs plaident en faveur d'une sédentarisation (voie d'accès à la reconnaissance dans le contexte de la nouvelle politique de décentralisation), séduisant les fractions les plus pauvres autour de l'installation des Postes de Santé Avancés, alors que, sur le plan sanitaire, la sédentarisation n'est pas forcément la meilleure option pour les nomades. Les élites touareg "modernisées" tentent ainsi de combiner les avantages de la sédentarisation avec le maintien des agriculteurs et des pasteurs les plus pauvres dans une situation de dépendance. Plus ça change...

7 La contribution de Mamadou Sall illustre plus spécifiquement deux aspects: la "projection spatiale de l'architecture sociale" sur le terroir et la maximalisation des profits que produit la démultiplication des statuts. La projection vise à assurer la reproduction du système et sa viabilisation. Dans la vallée du fleuve Sénégal, la duplication ancienne de l'organisation spatiale complexe de la rive gauche vers la rive droite du fleuve visait à réduire les risques et donc à accroître la viabilité du système. La situation créée par le conflit sénégalo-mauritanien a amené l'intervention des grands organismes humanitaires et l'établissement de nouveaux statuts gérant l'accès à 
leurs ressources. Dans la région un ensemble de pratiques s'est développé en vue d'assurer l'accès aux ressources et services publics des deux côtés de la frontière.

Ces stratégies impliquent, de façon analogue à celles que déploient les migrants, de tirer bénéfice de la transhumance en recourant à des assises sociales multiples. La trajectoire que décrit $\mathrm{S}$. Bava en est l'illustration. Cette trajectoire met en évidence les rituels administratifs qui assurent le passage de registre. En contraste, le texte de Simon Turner illustre le rituel du HRC consistant à "mettre à plat" et à uniformiser la population d'un camp de réfugiés burundais en Tanzanie. Quelle que soit la puissance de cette apparente uniformisation, les réfugiés se réapproprient la situation, la plient à leurs habitus. Dans ce cas précis, la hiérarchisation de la société est reconstruite par la rumeur. Les bruits relatifs à des vols, à des affiliations politiques, les violences et les craintes de violence recréent du pouvoir et des inégalités là où toutes les structures spatiales et les distributions de ressources ont été égalisées.

Me souvenant des vingt-six camps de réfugiés rwandais de Bukavu où, dans certains cas, l'aménagement sauvage de l'espace par les réfugiés reproduisait la projection sociale dans l'espace collinaire rwandais, la ré-appropriation socio-culturelle de l'espace et de la gestion des autres ressources des camps me semble l'élément essentiel à retenir pour arrimer les textes de cet atelier au thème général du colloque. Tout comme dans les projets de développement - ou d'équipement - les nouvelles ressources mises à la disposition, et notamment les services publics, voient leur sens et leurs bénéfices canalisés en fonction des habitus locaux et des stratégies sociales possibles. L'introduction de nouvelles normes relatives à l'installation et à l'usage de ces services crée une situation de pluralisme de références et élargit le registre d'intervention des acteurs. Les stratégies visent à maximaliser et à pérenniser les acquis de l'adaptation réussie à une nouvelle configuration du milieu. L'ensemble de ces textes gagne à être mis en perspective avec la contribution de Marion Fresia (publiée dans ce numéro). L'hybridité des approches de l'humanitaire qu'elle y décrit dans le contexte sénégalo-mauritanien est un phénomène général et, par essence, multiforme. Cette re-appropriation locale s'insère, à plus long terme, dans deux logiques : une logique d'étatisation des services publics humanitaires, une logique endogène de factionnalisme et de politisation des services.

\section{AUTEURS}

\section{DANIELLE DE LAME}

Musée royal de l'Afrique centrale, Leuvensesteenveg 13. 3080 Tervuren, Belgique, tel. : (+32) 27695247 ; fax. : (+32) 27695642 ; danielle.delame@skynet.be

\section{WIN VAN BINSBERGEN}

African Studies Centre, Leiden, tel. : (+31) 0715273677 ; binsbergen@fsw.leidenuniv.nl 